\title{
$O$ enfermeiro na qualidade e segurança do paciente
}

The nurse in patient quality and safety

La enfermera en calidad y seguridad del paciente

Bianca Campos de Oliveira

ORCID: https://orcid.org/0000-0001-8144-8453

Universidade do Estado do Pará, Brasil

E-mail: biancaoliveira96142@gmail.com

Katielem Melo Vale

ORCID: https://orcid.org/0000-0003-4868-1223

Centro Universitário Metropolitano da Amazônia, Brasil

E-mail: katielem.enf@gmail.com

Milena Farah Damous Castanho Ferreira

ORCID: https://orcid.org/0000-0002-0645-2046

Centro Universitário Metropolitano da Amazônia, Brasil

E-mail: milenafcastanho@hotmail.com

Brenda Tanielle Dutra Barros

ORCID: https://orcid.org/0000-0002-3184-050X

Centro Universitário Metropolitano da Amazônia, Brasil

E-mail: brendatanielle.enf@gmail.com

Rômulo Leno Miranda Barros

ORCID: https://orcid.org/0000-0003-1802-4521

Universidade da Amazônia, Brasil

E-mail: romuloleno@hotmail.com

Virgínia Mercês Lara Pessoa Oliveira

ORCID: https://orcid.org/0000-0002-4417-637X

Centro Universitário Metropolitano da Amazônia, Brasil E-mail: virginiaoliveira@famaz.edu.br

Camila Negrão Monteiro

ORCID: https://orcid.org/0000-0003-0972-5879

Universidade Federal do Pará, Brasil

E-mail: kcalnegrao@yahoo.com.br

Clarissa Porfírio Mendes

ORCID: https://orcid.org/0000-0001-6488-718X

Universidade do Estado do Pará, Brasil

E-mail: clarissapmendes@yahoo.com.br

Taís dos Passos Sagica

ORCID: https://orcid.org/0000-0002-6871-0100

Universidade Federal do Pará

E-mail: thaispassos12@gmail.com

Nancy de Souza Felipe de Nazaré

ORCID: https://orcid.org/0000-0003-1408-9598

Universidade da Amazônia, Brasil

E-mail: nancy_felipe2007@yahoo.com.br

Kátia Cilene Lisboa Farias

ORCID: https://orcid.org/0000-0001-7667-7163

Fundação Santa Casa de Misericórdia do Pará, Brasil

E-mail: nunescilenelisboa@gmail.com

Victória Karolina Santos Santana

ORCID: https://orcid.org/0000-0003-2379-8353

Universidade do Estado do Pará, Brasil E-mail: vickarolsantana@gmail.com

Tatyanne Lima Rocha Ferreira

ORCID: https://orcid.org/0000-0002-5071-1439 Universidade do Estado do Pará, Brasil

E-mail: tatyannelima16@gmail.com

João Lucas Moraes Souza

ORCID: https://orcid.org/0000-0003-3652-751X Universidade do Estado do Pará, Brasil

E-mail: jlucasmsouza@gmail.com

Tatiana Fabíola da Silva Lima

ORCID: https://orcid.org/0000-0001-5966-9727 Universidade do Estado do Pará, Brasil E-mail: tatiana.fdslima@aluno.uepa.br 


\begin{abstract}
Resumo
Na enfermagem, o cuidado e segurança do paciente é um fator primordial desde os tempos de Florence Nightingale e muitos pesquisadores acreditam que as teorias ajudam na obtenção de melhores resultados na enfermagem. O objetivo da pesquisa foi conhecer as evidências científicas a respeito dos processos de trabalho que englobam a segurança do paciente e o gerenciamento de risco. Percurso metodológico do tipo Revisão Integrativa de Literatura, através do modelo descritivo, com abordagem qualitativa. Para análise, foi utilizada a "Análise do Conteúdo Temático de Bardin”. Para coleta de dados, foi utilizado um instrumento adaptado de (Ursi 2005). Para busca científica foram selecionados artigos referentes ao período de 2016 a 2020, que tinham como descritores: "Enfermagem"; "Gestão de qualidade"; "Segurança do paciente". Foram buscados os acervos nas bases de dados da LILACS e SCIELO. A amostra final foi composta por 12 artigos científicos, sendo que nos resultados houve maior concentração na base de dados SCIELO com 7 artigos, correspondendo a $58 \%$ e no LILACS com 5 artigos, correspondendo a $42 \%$ do total da amostra. Discutiu-se sobre como são desempenhados os processos de trabalho realizados por enfermeiros e como a sua percepção é importante no pensar e tomar decisões. As relações diárias com membros de outras áreas de atuação para almejar propósitos através das capacitações, ciclos de melhorias nos leva a identificar o sistema social nessas atividades, que além de uma interação dinâmica, leva esses profissionais a trabalhar em conjunto em prol de um bem comum: a segurança do paciente.
\end{abstract}

Palavras-chave: Enfermagem; Gestão de qualidade; Segurança do paciente.

\begin{abstract}
In nursing, patient care and safety has been a key factor since Florence Nightingale's time and many researchers believe that theories help to achieve better nursing outcomes. The objective of the research was to know the scientific evidence regarding the work processes that encompass patient safety and risk management. Methodological path of the Integrative Literature Review type, through the descriptive model, with a qualitative approach. For analysis, the "Thematic Content Analysis of Bardin" was used. For data collection, an instrument adapted from (URSI 2005) was used. For scientific search, articles referring to the period from 2016 to 2020 were selected, which had as descriptors: "Nursing"; "Quality management"; "Patient safety". The collections were searched in LILACS and SCIELO databases. The final sample consisted of 12 scientific articles, and the results were more concentrated in the SCIELO database with 7 articles, corresponding to 58\%, and in LILACS with 5 articles, corresponding to $42 \%$ of the total sample. It was discussed how the work processes performed by nurses are performed and how their perception is important in thinking and making decisions. The daily relationships with members from other areas of expertise to pursue purposes through training, improvement cycles leads us to identify the social system in these activities, which in addition to a dynamic interaction, leads these professionals to work together for a common good: patient safety.
\end{abstract}

Keywords: Nursing; Quality management; Patient safety.

\title{
Resumen
}

En enfermería, el cuidado y la seguridad del paciente ha sido un factor clave desde la época de Florence Nightingale y muchos investigadores creen que las teorías ayudan a lograr mejores resultados de enfermería. El objetivo de la investigación fue conocer la evidencia científica sobre los procesos de trabajo que engloban la seguridad del paciente y la gestión de riesgos. Ruta metodológica del tipo Revisión Integrativa de la Literatura, a través del modelo descriptivo, con enfoque cualitativo. Para el análisis se utilizó el "Análisis de contenido temático de Bardin". Para la recopilación de datos, se utilizó un instrumento adaptado de (URSI 2005). Para la búsqueda científica se seleccionaron artículos referidos al período de 2016 a 2020, los cuales tenían como descriptores: "Enfermería"; "Gestión de la calidad"; "Seguridad del paciente". Las colecciones se buscaron en las bases de datos LILACS y SCIELO. La muestra final estuvo conformada por 12 artículos científicos, y los resultados estuvieron más concentrados en la base de datos SCIELO con 7 artículos, correspondiente al 58\%, y en LILACS con 5 artículos, correspondiente al $42 \%$ de la muestra total. Se discutió cómo se realizan los procesos de trabajo que realizan las enfermeras y cómo su percepción es importante para pensar y tomar decisiones. La relación diaria con miembros de otras áreas de especialización para perseguir propósitos a través de la formación, ciclos de mejora nos lleva a identificar el sistema social en estas actividades, que además de una interacción dinámica, lleva a estos profesionales a trabajar juntos por un bien común: la seguridad del paciente.

Palabras clave: Enfermería; Gestión de la calidad; Seguridad del paciente.

\section{Introdução}

A qualidade na saúde vem sendo discutido há tempos, e tem como princípio a obtenção do maior benefício para o paciente, ao menor custo e com o menor risco possível. Avedis Donabedian foi um médico pioneiro na discussão sobre essa temática, sua abordagem se deu através de três elementos conhecidos como a tríade Donabediana: estrutura, processos e resultados, que juntamente com as dimensões da qualidade: Eficácia, Eficiência, Efetividade, Otimização, Aceitabilidade, 
Legitimidade e Acessibilidade, são caracterizados como os sete pilares da qualidade, foi também mais uma contribuição de Donabedian que teve como intuito inicial mensurar a qualidade em saúde (Brasil, 2019).

Falar da enfermagem, é falar de segurança do paciente, a Organização Mundial de Saúde estima que anualmente milhares de pessoas em diversos países, sofrem danos causados pela ocorrência de eventos adversos (EA) a maioria dos erros é relacionado à sobre carga de trabalho, repetição de tarefas, falta de capacitação do pessoal e ausência de barreiras de segurança eficazes. Para melhor identificação dos tipos de consequências para o paciente, os incidentes foram estratificados e classificados como: circunstância de risco (reportable circums - tance), quase incidente (near-miss), incidente sem danos (no harm incident) ou incidente com dano (harmful incident), que são referidos também como evento adverso EA (adverse event) (Françolin et al., 2015).

Por estes motivos a portaria de no 529 e 941/2013 vem discorrer sobre a Política Nacional de Segurança do Paciente (PNSP), lançada pelo Ministério da Saúde (MS) e tem seus objetivos baseados em promover ações com foco na segurança do paciente através da implantação dos Núcleos de Segurança do Paciente, que passou a ser obrigatório em todas as instituições de saúde pela RDC No 36 e define a PNSP como um conjunto de atributos nos serviços de saúde, desenvolvida para promover e incentivar a adoção de ações voltas para à segurança do paciente (Brasil, 2013)

Uma das medidas para se alcançar padrões de excelência na assistência ao paciente foi a implantação dos 6 (seis) protocolos das metas padrão da segurança do paciente: Identificação do paciente; Comunicação efetiva; Segurança na prescrição e administração de medicamentos; Cirurgia segura; Prática de higienização das mãos em serviços de saúde; Prevenção de quedas e lesão por pressão. É a partir do reconhecimento de potenciais e fragilidades que surgem oportunidades de crescimento e resultados cada vez mais positivos as instituições de saúde e aos pacientes (Forte et al., 2018).

Muitos pesquisadores afirmam que as teorias ajudam na obtenção de melhores resultados na enfermagem, e para isso precisam ser revisadas e analisadas por sua relevância, aplicação na prática, e na administração da enfermagem para que sejam inseridas de forma integral. Tais teorias tem uma grande relevância em todas as dimensões do cuidado, contribuindo no aprofundamento da enfermagem como um todo, e nesse cenário, enfermeiras teóricas foram se destacando, Imogene King foi uma delas (Neto et al., 2016).

O gerenciamento de risco (GR) é a aplicabilidade sistemática de políticas de gestão, procedimentos, tomadas de decisões e ações que servem para analisar, controlar e monitorar o risco de ocorrências de eventos adversos de maneira sistemática e contínua. Nesse contexto, o enfermeiro gestor se torna um intermediário facilitador e motivador, na busca do envolvimento de todos no processo de alcance aos resultados positivos na prestação da assistência segura. No Brasil se teve a expansão da GR através da Agência Nacional de Vigilância Sanitária (ANVISA) no projeto Hospitais Sentinelas. (Siqueira, Silva, Teles, Feldman, 2015).

O enfermeiro é o profissional que está na base da assistência, e como gestor é também educador, tem a competência de monitorar mais de perto os procedimentos de cuidados prestados aos pacientes. Para os profissionais da enfermagem o acontecimento de um EA pode subsidiar diversos problemas, que vão de dano ao paciente, estresse emocional a punições legais. Tendo conhecimento disso, o investimento em ferramentas que ajudem na melhora da qualidade da assistência, fomentem a implantação da cultura de segurança, atuem na identificação de situações de risco, análise, localização e resolução de eventuais erros é de extrema importância, uma dessas ferramentas é notificações de eventos, que também possibilita a equipe assistencial compartilhar responsabilidades com os administradores e melhorar a comunicação entre setores, processo que pode ser monitorado e administrado pelo GR (Paiva et al., 2014).

Como citado anteriormente, teorias de enfermagem são embasadas em evidências científicas, e avaliadas quanto sua relevância para sociedade, desse modo podem ser descritas como: 
Uma articulação organizada, coerente e sistemática de um conjunto de afirmações relacionadas as perguntas significativas de uma disciplina, comunicadas, compartilhadas em conjunto em um todo significativo, com o objetivo de descrever os fenômenos, explicar as relações entre eles e predizer consequências ou prescrever o cuidado de enfermagem. Nesse contexto, tais teorias se desenvolvem com a finalidade de refletir os interesses da comunidade cientifica e da sociedade (Neto et al., 2016, p.175).

O estudo é relevante, pois pretende construir conhecimento acerca da atuação do enfermeiro da qualidade e os processos que visam garantir um bom gerenciamento e consequentemente a segurança do paciente e como se enquadra nas suas atividades diárias.

A relevância de tratar sobre segurança do paciente e a importância do enfermeiro frente o gerenciamento de risco e seus processos, é proporcionar informação aos futuros profissionais da saúde sobre a importância que a segurança do paciente e o seu gerenciamento representam para uma assistência à saúde digna e de qualidade. A questão norteadora da pesquisa embasa se em quais as evidências na literatura sobre segurança do paciente e a importância do enfermeiro frente o gerenciamento de risco e seus processos no período de 2016 à 2020 ?

O objetivo dessa pesquisa é conhecer as evidências científicas no que desrespeito os processos de trabalho que englobam a segurança do paciente e o gerenciamento de risco.

\section{Metodologia}

O estudo foi do tipo Revisão Integrativa de Literatura (RIL), através do modelo descritivo, com abordagem qualitativa.

A RIL possui seis fases, sendo elas: 1 fase: elaboração da pergunta norteadora. 2 fase: busca ou amostragem na literatura. 3 fase: coleta de dados. 4 fase: análise crítica dos assuntos incluídos. 5 fase: discussão dos resultados. 6 fase: apresentação da revisão integrativa (Souza, Silva \& Carvalho, 2010, p 103).

Para busca científica foram selecionados artigos sobre o objeto de estudo, referentes ao período de 2016 a 2020, que tinham como descritores: "Enfermagem"; "Gestão de qualidade"; "Segurança do paciente". Foram buscados os acervos nas bases de dados da Literatura Latino-amaricana, do Caribe em Ciências da Saúde (LILACS), e Scientific Electronic Library Online (SCIELO).

Utilizou-se como critérios de inclusão artigos completos disponíveis em língua portuguesa que abordassem a temática escolhida e que estivessem no período definido para o estudo. Como critérios de exclusão optou-se por não utilizar textos em língua estrangeira, publicações fora do período de estudo e que não possuem aproximação temática, assim como, tese, dissertação, manuais e protocolos.

Para coleta de dados, foi utilizado um instrumento adaptado de (Ursi 2005). Neste instrumento, após leitura exaustiva dos artigos, os dados extraídos foram transcritos, possibilitando o detalhamento de cada estudo, podendo ser organizados por planilhas em ordem numérica crescente, no programa Microsoft Excel.

Para análise foi utilizada a "Análise do Conteúdo Temático de Bardin (ACTB)", cujo objetivo é compreender o sentido das comunicações e suas significações explícitas e/ou ocultas. Segundo Bardin (2009), a análise de conteúdo resume-se a um conjunto de técnicas de análises das comunicações, visando obter, por procedimentos sistemáticos e objetivos de descrições de mensagens, indicadores que permitam a inferência de conhecimentos relativos às condições de produção/ recepção destas mensagens.

As análises de estudo foram apresentadas na seguinte sequência: descrição de dados de identificação das publicações (autores e ano), do Estado e instituição sede do estudo e do tipo de revista científica; e a seguir organizados em um quadro, que descreverá as características metodológicas dos estudos, classificando-os de acordo com o delineamento de pesquisa e nível de 
evidências; descrição da análise propriamente dita, agrupada em categorias ou temas.

\section{Resultados}

Inicialmente, foram identificados 132 artigos encontrados a partir dos descritores preestabelecidos nas bases de dados pesquisadas. Dos artigos alcançados, 47 encontravam-se no LILACS e 85 no SCIELO. Com o manuseio para exclusão das duplicatas, restaram 54 trabalhos que tiveram seus resumos lidos e analisados, objetivando confirmar se tratavam da abordagem sobre Segurança do paciente e o gerenciamento de risco em eventos adversos relacionados à assistência à saúde. Com a leitura crítica e a utilização das etapas de Bardin, somados a aplicação dos critérios de inclusão e exclusão resultou em uma amostra final de 12 artigos.

A amostra final desta revisão foi composta por 12 artigos científicos, sendo que nos resultados houve maior concentração na base de dados SCIELO com 7 artigos, correspondendo a 58 \% e no LILACS com 5 artigos, correspondendo a $42 \%$ do total da amostra.

Os estudos que, ao serem selecionados e após leitura crítica não atenderam ao período definido de publicação ou que, em seu título fizessem as referências solicitadas e de alguma forma remetesse às mesmas, porém em seus resultados não se tenha evidenciado a relação entre a Segurança do paciente e os gerenciamentos de risco em eventos adversos relacionados à assistência à saúde, foram devidamente excluídos.

De acordo com os resultados obtidos, observasse-se que a segurança do paciente é a qualidade do serviço prestado. As produções científicas acerca dessa categoria destacam a relevância da avaliação dos cuidadores pela equipe de enfermagem, para melhorar a qualidade da assistência prestada ao paciente.

Entretanto os estudos destacam que para melhorar a atuação do enfermeiro no contexto hospitalar, é necessário que haja compromisso das lideranças para difundir a necessidade de mudança de comportamento e de cultura. No sentido de promover a superação da visão restrita dos profissionais de saúde o líder deve utilizar-se de estratégias como a interdisciplinaridade e a educação permanente, com o intuito de resgatar a produção do conhecimento por meio da vivência, da parceria e da indissociabilidade entre teoria e prática (Silva et al., 2018).

A partir da análise do estudo, emergiram duas categorias: papel do enfermeiro no controle de eventos adversos e desafios da equipe de enfermagem acerca da Segurança do paciente.

Para a melhor compreensão de como os estudos foram incluídos nesta revisão, os dados obtidos foram dispostos na tabela a seguir:

Tabela 1. Descrição dos estudos incluídos na revisão integrativa, segundo base de dados, títulos, autores, objetivos e ano de publicação e periódicos.

\begin{tabular}{|c|c|c|c|c|c|}
\hline $\begin{array}{l}\text { BASE } \\
\text { DE } \\
\text { DADOS }\end{array}$ & TITULO & AUTORIA & OBJETIVOS & $\begin{array}{c}\text { ANO DE } \\
\text { PUBLICAÇÃO }\end{array}$ & PERIÓDICO \\
\hline SCIELO & $\begin{array}{c}\text { Posicionamento de gestores e } \\
\text { lideranças de enfermagem } \\
\text { diante dos erros divulgados } \\
\text { na mídia. }\end{array}$ & Elaine, C.N.F; et al. & $\begin{array}{l}\text { Analisar o posicionamento de } \\
\text { gestores e lideranças } \\
\text { enfermagem acerca dos erros } \\
\text { enfermagem divulgados na mídia. }\end{array}$ & 2018 & $\begin{array}{l}\text { REVISTA } \\
\text { GAÚCHA } \\
\text { ENFERM. }\end{array}$ \\
\hline SCIELO & $\begin{array}{l}\text { Avaliação da cultura de } \\
\text { segurança do paciente: } \\
\text { estudo comparativo em } \\
\text { hospitais universitário }\end{array}$ & Cinita, S.F; et al & $\begin{array}{l}\text { Realizar uma comparação dos } \\
\text { compósitos da cultura de segurança } \\
\text { do paciente com base na avaliação } \\
\text { de enfermeiros brasileiros e } \\
\text { portugueses que trabalham em } \\
\text { hospitais universitários. }\end{array}$ & 2018 & $\begin{array}{c}\text { REV ESC } \\
\text { ENFERM USP. }\end{array}$ \\
\hline SCIELO & $\begin{array}{c}\text { Cultura de segurança do } \\
\text { paciente: avaliação pelos } \\
\text { profissionais de enfermagem }\end{array}$ & Danieli, B.C; et al. & $\begin{array}{l}\text { Avaliar a cultura de segurança do } \\
\text { paciente das equipes de } \\
\text { enfermagem no contexto hospitalar. }\end{array}$ & 2018 & $\begin{array}{l}\text { TEXTO } \\
\text { CONTEXTO } \\
\text { ENFERM }\end{array}$ \\
\hline
\end{tabular}




\begin{tabular}{|c|c|c|c|c|c|}
\hline SCIELO & $\begin{array}{l}\text { Escala de eventos adversos } \\
\text { associados às práticas de } \\
\text { enfermagem: estudo } \\
\text { psicométrico em contexto } \\
\text { hospitalar }\end{array}$ & Tereza, N.; et al. & $\begin{array}{l}\text { Contribuir para o estudo de } \\
\text { validação da Escala de Eventos } \\
\text { Adversos associados às Práticas de } \\
\text { Enfermagem, em contexto } \\
\text { hospitalar. }\end{array}$ & 2018 & $\begin{array}{l}\text { REV. LATINO- } \\
\text { AM. } \\
\text { ENFERMAGEM }\end{array}$ \\
\hline SCIELO & $\begin{array}{l}\text { A prática de notificação de } \\
\text { eventos adversos em um } \\
\text { hospital de ensino }\end{array}$ & $\begin{array}{l}\text { Andréia, G.S; SImone, } \\
\text { G.S.C; Maria, J.M.B }\end{array}$ & $\begin{array}{l}\text { Compreender a prática de relatar } \\
\text { eventos adversos por profissionais } \\
\text { de saúde. }\end{array}$ & 2017 & $\begin{array}{c}\text { REV ESC } \\
\text { ENFERM USP. }\end{array}$ \\
\hline SCIELO & $\begin{array}{l}\text { Gravidade e carga de } \\
\text { trabalho relacionadas a } \\
\text { eventos adversos em UTI }\end{array}$ & Clarita, T.R.S; et al. & $\begin{array}{l}\text { Analisar se o aumento da gravidade } \\
\text { do paciente e a carga de trabalho de } \\
\text { enfermagem está relacionado à } \\
\text { maior incidência de Eventos } \\
\begin{array}{l}\text { Adversos (EAs) em pacientes } \\
\text { críticos. }\end{array}\end{array}$ & 2017 & $\begin{array}{l}\text { REV BRAS } \\
\text { ENFERM. }\end{array}$ \\
\hline SCIELO & $\begin{array}{l}\text { Percepção da enfermagem } \\
\text { frente ao clima de segurança } \\
\text { do paciente em instituições } \\
\text { públicas e privadas }\end{array}$ & Renata, C.G; et al. & $\begin{array}{l}\text { Avaliar a percepção da equipe de } \\
\text { enfermagem frente ao clima de } \\
\text { segurança que permeia a assistência } \\
\text { entre profissionais de instituições } \\
\text { públicas e privadas. }\end{array}$ & 2017 & $\begin{array}{l}\text { REV GAÚCHA } \\
\text { ENFERM }\end{array}$ \\
\hline LILACS & $\begin{array}{l}\text { A cultura de segurança do } \\
\text { paciente no âmbito da } \\
\text { enfermagem: reflexão teórica }\end{array}$ & Graziele, C.L; et al. & $\begin{array}{l}\text { Refletir sobre o conceito de cultura } \\
\text { de segurança e suas dimensões, no } \\
\text { contexto da equipe de enfermagem. }\end{array}$ & 2018 & $\begin{array}{l}\text { REVISTA DE } \\
\text { ENFERMAGEM } \\
\text { DO } \\
\text { CENTROOESTE } \\
\text { MINEIRO }\end{array}$ \\
\hline LILACS & $\begin{array}{c}\text { Cultura de segurança do } \\
\text { paciente em hospital privado }\end{array}$ & Lidiane, G. et al. & $\begin{array}{l}\text { Avaliar a percepção dos } \\
\text { profissionais de enfermagem } \\
\text { atuantes em um hospital privado } \\
\text { acerca do clima de segurança. }\end{array}$ & 2018 & $\begin{array}{c}\text { J. RES.: } \\
\text { FUNDAM. } \\
\text { CARE. ONLINE. }\end{array}$ \\
\hline LILACS & $\begin{array}{l}\text { Cultura de segurança do } \\
\text { paciente em centro cirúrgico: } \\
\text { percepção da equipe de } \\
\text { enfermagem }\end{array}$ & $\begin{array}{l}\text { Elena Bohomol , Eliana } \\
\text { Ferreira de Melo. }\end{array}$ & $\begin{array}{l}\text { Analisar a percepção de } \\
\text { profissionais de enfermagem de um } \\
\text { centro cirúrgico em um hospital } \\
\text { privado acerca das dimensões da } \\
\text { cultura de segurança do paciente. }\end{array}$ & 2019 & REV. SOBECC \\
\hline LILACS & $\begin{array}{l}\text { Segurança do paciente na } \\
\text { visão de enfermeiros: uma } \\
\text { questão multiprofissional }\end{array}$ & $\begin{array}{l}\text { Marcos Antônio Nunes } \\
\text { de Araújo, Wilson } \\
\text { Danilo Lunardi Filho, } \\
\text { Rosemary Silva da } \\
\text { Silveira, José Carlos } \\
\text { Souza, Edsom Luís } \\
\text { Devos Barlem, Nanci da } \\
\text { Silva Teixeira }\end{array}$ & $\begin{array}{l}\text { Identificar como o enfermeiro } \\
\text { percebe a segurança do paciente na } \\
\text { instituição de saúde em que atua. }\end{array}$ & 2017 & ENFERM. FOCO \\
\hline LILACS & $\begin{array}{l}\text { Protocolos Operacionais } \\
\text { Padrão na prática profissional } \\
\text { da enfermagem: utilização, } \\
\text { fragilidades e potencialidades }\end{array}$ & Camila, B.S; et al. & $\begin{array}{l}\text { Avaliar a utilização dos } \\
\text { Procedimentos Operacionais Padrão } \\
\text { (POP) na prática profissional da } \\
\text { equipe de enfermagem, com base } \\
\text { no referencial teórico de } \\
\text { Donabedian, bem como identificar } \\
\text { as fragilidades e potencialidades a } \\
\text { partir da sua implantação. }\end{array}$ & 2017 & $\begin{array}{l}\text { REV BRAS } \\
\text { ENFERM }\end{array}$ \\
\hline
\end{tabular}

Fonte: Autores.

\section{Discussão}

Na primeira categoria, observa-se nos estudos selecionados que; para o profissional de enfermagem, a ocorrência dos eventos adversos pode acarretar diversas problemáticas, dado o estresse emocional, os preceitos éticos e às punições legais a que está exposto. Assim, é importante o investimento numa cultura de segurança, por meio da disseminação do conceito de segurança do paciente e de uma discussão não punitiva sobre os eventos adversos (Silva et al., 2018).

O Gerenciamento de risco é fundamental em relação aos Eventos adversos (EA), as ocorrências são perigosas tanto para o paciente quanto para os profissionais de saúde, pois acarretam sofrimentos, longa permanência na instituição e com isso gastos excessivos, a adoção rotineira das precauções-padrão, previnem que erros desnecessários ocorram. O GR visa minimizar os danos relacionados, uma vez que prioriza o aprendizado com falhas e a prevenção de novo incidente relacionada à assistência à saúde (Fassarella et al., 2018). 
O número adequado de profissionais é indispensável para o cuidado seguro, sendo responsabilidade institucional prover condições favoráveis de recursos humanos nas unidades. Afinal, a adequação quantitativa de profissionais, segundo as necessidades dos pacientes, pode possibilitar não só menor risco aos pacientes como também menor incidência de agravos à saúde dos trabalhadores (Silva et al., 2018).

O Gerenciamento de risco desenvolve um trabalho analítico e crítico, podendo servir também para o balanço dos indicadores de processos da enfermagem, pois os indicadores são informações importantes que expressam o desempenho de um processo sendo o mais objetivo possível. A constante busca para uma assistência de qualidade, segura e com a satisfação dos usuários precisa ser monitorado e avaliado periodicamente, través dos padrões que já estão pré-estabelecidos, esses serviços podem ser avaliados através da acreditação (Reis et al., 2017).

Os estudos selecionados relatam a redução e gerenciamento de erros na área da saúde através de trabalho em equipe executado com qualidade e cooperação de todos no processo. Neste sentido, observa-se que os cuidados de saúde modernos estão cada vez mais baseados em equipes multidisciplinares que se organizam numa rede mais complexa e compartilhada.

$\mathrm{Na}$ segunda categoria, observa-se que os estudos descrevem que a segurança do paciente representa um dos maiores desafios para a excelência da qualidade no serviço de saúde. O ambiente, as tarefas, a organização e a tecnologia são elementos do sistema de trabalho que interferem na qualidade da assistência prestada ao paciente. As condições de trabalho são fatores que comprometem a qualidade do cuidado (Araújo et al., 2017).

Atualmente a cultura de segurança está se tornando alvo de atenção e prioridade internacional e é compreendida como o somatório de valores, vivências, costumes e práticas que definem o comportamento de um grupo. Para ser alcançada, essa cultura requer um conhecimento de valores, crenças e normas sobre o que é importante em uma instituição e que atitudes e comportamentos são esperados. Estabelecer uma cultura de segurança é o elemento chave de instituições de alta confiabilidade (Siman, Cunha \& Brito, 2017).

O papel da liderança é uma componente chave para o desenvolvimento de uma cultura de segurança, pois por meio da atuação de um líder, os demais profissionais conseguem criar estratégias e estruturas para promover cuidados de saúde seguros e com qualidade. Além disso, ajudam a moldar uma cultura em que os erros e as falhas sejam vistos como uma forma de conhecimento e aprendizagem contínua (Lemos et al., 2018).

Por isso, a cultura de segurança tem papel fundamental para a definição de comportamentos e implementações de ações que visem à melhoria da qualidade institucional, como a utilização dos 6 (seis) protocolos das metas padrão da segurança do paciente por exemplo. É a partir do reconhecimento de potenciais e fragilidades que surgem oportunidades de crescimento e resultados cada vez mais positivos, tanto para os pacientes quanto para as instituições de saúde (Forte et al, 2018).

Uma conexão entre a cultura de segurança e os resultados na assistência prestada ao paciente precisam estar eminentes para se obter resultados positivos, não se esquecendo da liderança que a proposito corresponde a competência mais importante nos gerentes de enfermagem, e terá um papel de suma importância no processo, pois com uma boa atuação dos demais profissionais é possível desenvolver planos e estruturas que fortaleçam os cuidados de saúde com qualidade. Sabendo das incertezas e imprevistos de um panorama assistencial, se faz necessário que a liderança e gerentes sejam extremamente conhecedores das fragilidades e potencialidades de sua equipe (Lemos, et al, 2018).

\section{Conclusão}

Diante a análise das categorias, foi possível discutir sobre como são desempenhados os processos de trabalho realizados por enfermeiros, como a sua percepção é importante no pensar e tomar decisões, como demonstrado na análise indicadora. Relações diárias com membros de outras áreas de atuação para almejar propósitos através das capacitações, ciclos 
de melhorias, o que nos leva a identificar o sistema social nessas atividades, que além de uma interação dinâmica, esses profissionais irão trabalhar em conjunto em prol de um bem comum, a segurança do paciente.

Destacando assim que a enfermagem é considerada como um dos pilares principais para o gerenciamento da segurança do paciente, reforçando a necessidade de qualificação profissional, para a tomada de decisão na estratégia da instituição, a partir da evidência sobre a importância do gerenciamento, no sentido de adotar medidas para a segurança do paciente.

Contudo, faz-se necessário que o profissional enfermeiro sinta-se qualificado e busque sempre a atualização dos seus conhecimentos para aprimorar os processos de gerenciamento nas unidades de saúde visando assim, sempre a segurança e o bem-estar do cliente, da comunidade e do próprio profissional.

\section{Referências}

Araújo, I. M. de A., Oliveira, M. V. de \& Fernades, A. F. C. Compreensão do modelo de King sobre o paradigma do Interacionismo Simbólico Understanding. Revista Brasileira de enfermagem, 58(6), 715-718, 2005.

Báo, P, C, A. et al. Indicadores da qualidade: ferramenta para o gerenciamento de boas práticas em saúde. Revista Brasileira de Enfermagem, 2(72), 377-84. 2019.

Bardin, L. Analise de conteúdo. 70, 2011.

Brasil. Resolução no 466 e nº 510, de 7 de abril de 2016, do Conselho Nacional de Saúde, do Ministério da Saúde. Diário Oficial da União.

Brasil (ANVISA). Qualidade e segurança do paciente em serviços de saúde: noções gerais. Unidade 1. Curso de Segurança do Paciente e Qualidade em Serviços de Saúde, módulo 1. Brasília- DF: ANVISA, 2019. 〈https//www.escolavirtual.com.br〉.

Brasil, Ministério da Saúde. Agência Nacional de Vigilância Sanitária. Fundação Oswaldo Cruz. Anexo 01: Protocolo para a prática de higienização das mãos em serviços. <https://www20.anvisa.gov.br/segurancadopaciente/index.php/>.

Brasil, Agência Estadual de Vigilância Sanitária. ANVISA. Cartilha de segurança do paciente. 〈https://www.C:/User/Downloads/cartilha_agevisa-2.pdf >.

Brasil, Santa Casa de Misericórdia do Pará. <https://santacasa.pa.gov.br/missao-visao-valores/>.

Chaves, E. S., Araujo, T. L. \& Lopes, M. V. O. Clareza na utilização dos sistemas sociais da teoria de alcance de metas. Rev. da Esc. de Enfermagem, 41(4), 698-704, 2017.

Fontanella, B. J. B., Ricas, J. \& Turato, E. R. Amostragem por saturação em pesquisas qualitativas em saúde: contribuições teóricas. Cad. Saúde Pública, 24(1), 17-27.

Françolin, L. et al. Gerenciamento da segurança do paciente sob a ótica dos enfermeiros. Rev. Esc. Enf. USP, 49(2), 277-283, 2015.

Forte, E. C. N. et al. Posicionamento de gestores e lideranças de enfermagem diante dos erros divulgados na mídia. Revista Gaúcha de Enfermagem, 39, 1-9, 2018.

Gorge, A. G. \& Miyake I. D. Estudo comparativo das ferramentas para mapeamento das atividades executadas pelos consumidores em processos de serviço. Prod. 26(3)

Moura, E. R. F. \& Pagliuca, L. M. F. A teoria de King e sua interface com o programa Saúde da Família. Rev. Esc. Enf. USP, 38(3), $270-279,2004$.

Mantovani, F. M. Gerenciamento de caso como modelo de cuidado: reflexão na perspectiva da teoria de imogene king. Conselho Regional de Enfermagem do Paraná/ UFP. Revista Ciênc. Cuid. Saúde. Paraná, 2020.

Neto, J. M. R. et al. Análise de teorias de enfermagem de Meleis: revisão integrativa. Revista Brasileira de enfermagem, 69(1), 174-181, 2016.

Oliveira, J. L. C. \& Matsuda, L. M. Descredenciamento da certificação pela acreditação hospitalar: percepções de profissionais. Texto e Contexto Enfermagem, 25(1), 1-8, 2016.

Piovesan, A. \& Temporine, E. R. Pesquisa exploratória: procedimentos metodológicos para o estudo de fatores humanos no campo da saúde pública. Revista de saúde Pública, 29(4), 318-325, 1995

Piana, M. C. A pesquisa de campo. Editora UNESP; Cultura Acadêmica. 〈http://books.scielo.org〉.

Pissinate, C, S, P. Estabelecimento de metas no planejamento da aposentadoria: reflexão à luz de imogene king. Revista Min de Enfermagem, 24. 2020.

Ribeiro, O., Martins, M. M. F. P. S. \& Tronchin, D. M. R. Qualidade dos cuidados de enfermagem: um dos estudos em hospitais portugueses. Revista de Enfermagem Referência, 4(14), 89-100, 2017.

Santos, J. L. G. Governança da prática profissional de enfermagem no ambiente hospitalar. 2014. 253 f. Tese (doutorado em Enfermagem) - Programa de Pós-Graduação em Enfermagem, Universidade Federal de Santa Catarina, Florianópolis, 2014. 
Research, Society and Development, v. 10, n. 16, e47101616040, 2021

(CC BY 4.0) | ISSN 2525-3409 | DOI: http://dx.doi.org/10.33448/rsd-v10i16.16040

Silva, O, C. et al. A utilização do método PDCA para melhoria dos processos: um estudo de caso no carregamento de navios. Revista Espacios, 38(27), 9. Ano 2017

Silva, L, J. Aplicação das ferramentas da qualidade para melhoria de processos produtivos estudo de caso em um centro automotivo. Universidade de Taubaté, São Paulo, 2017. 〈http://ppga.com.br/aguiar_paulo_celso_golçalves.pdf>.

Teixeira, S. K. A. et al. Cuidado clínico de enfermagem à pessoa com Úlcera Venosa fundamentado na teoria de Imogene King. Revista Enfermagem Atual, 81(19), 31-37, 2019. 